\title{
Diversity Combining for Fast Frequency Hopping Multiple Access Systems Subjected to Nakagami- $m$ Fading
}

\author{
Sohail Ahmed, Lie-Liang Yang and Lajos Hanzo \\ School of ECS, University of Southampton, SO17 1BJ, UK. \\ Tel: +44-23-8059 3364, Fax: +44-23-8059 4508 \\ Email: \{sa03r,lly,lh\}@ees.soton.ac.uk; http://www-mobile.ees.soton.ac.uk
}

\begin{abstract}
The achievable performance of various diversity combining schemes used in fast frequency hopping (FFH) aided $M$-ary frequency shift keying (MFSK) systems operating in a multiple access scenario subjected to Nakagami- $m$ fading is investigated. Specifically, linear, selfnormalization, hard limiting majority vote, soft limiting, product combining and order statistics-normalized envelope detection based diversity combining schemes are considered. The comparison of various diversity combining schemes is based on the achievable bit error rate versus the number of simultaneous users supported. It is shown using simulation results that although some of the combining schemes considered result in an inferior performance compared to the optimum soft limiting combiner, they offer the advantage of achieving an acceptable interference suppression performance without requiring side information.
\end{abstract}

\section{INTRODUCTION}

Fast frequency hopping (FFH) based code division multiple access (CDMA) offers an attractive alternative to the direct sequence (DS) CDMA philosophy. Various researchers have shown that FFH aided CDMA exhibits advantages in terms of supporting a large number of users and offering a high spectral efficiency [1], [2]. The bit error rate (BER) performance of a single user in a multiple access (MA) system is dependent upon its capability to suppress the interference imposed by other users. The better is this interference suppression capability, the larger the number of users the system is capable of supporting. From the perspective of interference suppression, the choice of an appropriate diversity combining scheme is an important consideration in the design of a noncoherent FFH assisted $M$-ary frequency shift keying (MFSK) receiver. The conventional FFH-MFSK based MA system employs a hard-limiting diversity combiner in the receiver [1]. Based on the maximum likelihood criterion, Yue [3] derived an optimum combining scheme and approximated it with a soft limiting combiner, which operated on the principle of clipping the outputs of the energy detectors, if they exceeded a preset threshold, before performing linear combining. Yue [3] showed that the soft limiting combiner outperformed both the hard limiting combining and the linear combining schemes. In [4], [5], the optimum combiner derived in [3] was analyzed under Rician fading conditions and it was shown that even without setting accurate clipping thresholds, the achievable performance gain was substantial. However, adapting of the clipping thresholds at the energy detectors' output according to the Signal to Noise Ratio (SNR) is required for clipping at the most suitable level and thus side information is required in the FFH-MFSK receiver employing a soft limiting combiner.

In [6], the employment of various diversity combining schemes that do not require side information was considered in a MA scenario.

The financial support of the EPSRC, UK and EU under the auspices of the Phoenix and Newcom projects is gratefully acknowledged. The authors are also grateful to their colleagues for the enlightenment gained within the Phoenix consortium.
However, an explicit comparison of the schemes considered with the classic hard limiting [1] or the bench-mark soft limiting [3] combiner was not included in their contribution. Hence, in this paper, the achievable performance of various diversity combining schemes that may be used in FFH-MFSK systems for combatting the MA interference is investigated based on simulation results. Specifically, the hard limiting majority vote [1], the soft limiting [3], the selfnormalization [6], the product combining [7] and the order statisticsnormalized envelope detection [6] based diversity combining schemes will be considered. One of the most important performance parameters in the analysis of MA systems is the number of simultaneous users supported, since this determines the achievable spectral efficiency of the system, which is defined as the data rate supported per Hertz of bandwidth [2]. Thus, our metric for the comparison of the diversity combiners is the BER achieved, when a given number of users is supported. A Nakagami- $m$ fading channel is assumed, which is a realistic and flexible model of the mobile radio channel. It has been shown that although the product combining [7] and the order statistics-normalized envelope detection [6] based combiner result in an inferior performance in comparison to the optimum soft limiting combiner, they have the advantage of dispensing with any sideinformation and hence they constitute attractive design alternatives. The remainder of this paper is structured as follows. In Section II the system under consideration is described. Section III consists of a discussion of the various diversity combining schemes considered, while in Section IV the achievable performance is characterized. Finally, in Section V we present our conclusions.

\section{SYSTEM DESCRIPTION}

The system under consideration is a land mobile communication system consisting of a single cell serving multiple users and a basestation (BS), as considered in [3]. All the users communicate with each other through the BS, employing FFH-MFSK signals. The schematic of a FFH-MFSK transmitter is shown in Fig. 1. As shown in the figure, the MFSK signal modulates a carrier generated by a frequency synthesizer, which is controlled by a pseudo-noise (PN) generator. Each user is assigned a unique and random $L$-tuple address output by the PN generator, where $L$ is the number of frequency hops per symbol. The frequency of the hopped signal may assume any one of the $Q$ legitimate frequencies. It is assumed that we have $Q=M$, where $M$ is the modulation order. It has been shown [2] that the choice of $Q=M$ results in the best spectral efficiency. The hop or chip interval $T_{h}$ is related to the symbol interval $T_{s}$ by the relation $T_{h}=T_{s} / L$. Hence, if the data is transmitted at a rate of $R_{b}$ bits per second and $R_{s}$ is the symbol rate, then the hop rate is given by $R_{h}=R_{s} L=R_{b} L / b$, where $b=\log _{2} M$ is the number of bits per symbol. Furthermore, we assume that the bandwidth occupied by the signal transmitted during each FFH chip 


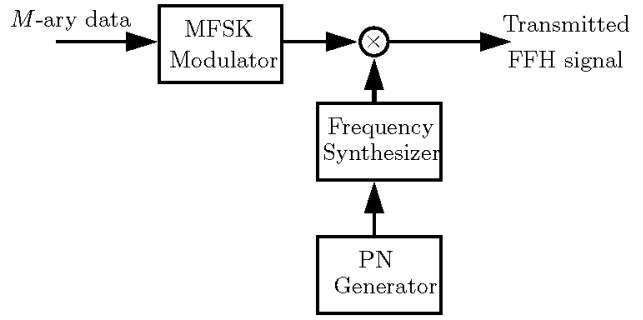

Fig. 1. Transmitter schematic of the FFH system using MFSK modulation

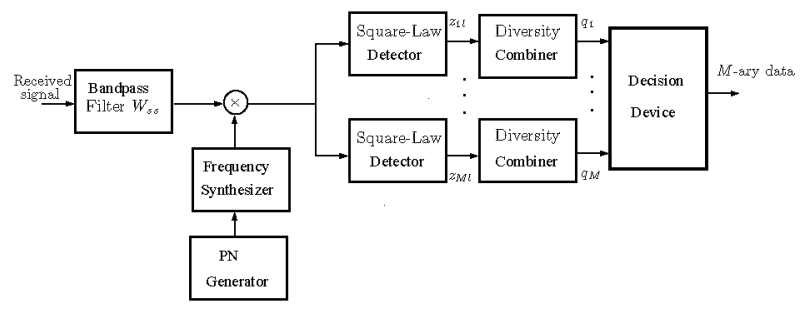

Fig. 2. Receiver schematic of the FFH-MFSK system using diversity combining

interval is approximated by that of its main spectral lobe occupying $R_{h}=1 / T_{h}$. The frequency separation between adjacent frequency hopping tones is also assumed to be $R_{h}$. Thus, the orthogonality of the FFH frequencies is maintained. Hence, we have,

$$
Q=\frac{W_{s s}}{R_{h}}=\frac{W_{s s} b}{R_{b} L} .
$$

The channel is modeled by Nakagami- $m$ fading for each of the transmitted frequencies. We assume that the frequency separation between the adjacent signalling frequencies is greater than the coherence bandwidth of the channel and thus, each signalling tone experiences independent fading. Moreover, the fading envelopes experienced by the various users are also independent of each other. However, the Nakagami fading parameter $m$ is the same for all the signalling tones of all the users.

Let $U$ be the total number of users supported by the communication system under consideration. Then, when a particular user transmits a signal to the BS, the signals transmitted by all other $(U-1)$ users can be modeled as continuous wave interfering tones. We assume a chip-synchronous system, where the chip transitions of all users are aligned. This may be achieved by the transmissions of periodic clock alignment signals from the BS to all users. Furthermore, perfect power control is assumed such that the power of all the signals received by the BS from all users is equal. Thus, the signal $r_{i}(t)$ recieved at the $\mathrm{BS}$ can be modeled as

$$
r_{i}(t)=h_{s} s_{i}(t)+\sum_{j=1}^{U^{\prime}} h_{j} I_{j}(t)+n(t)
$$

where $s_{i}(t)$ represents the desired signal transmitted by the $i$ th user, $i=1,2, \ldots, U, I_{j}(t)$ denotes the signal transmitted by the $j$ th interferer and $U^{\prime} \leq U-1$ is the number of active interferers. Furthermore, $h_{s}=\alpha_{s} e^{i \theta_{s}}$ and $h_{j}=\alpha_{j} e^{i \theta_{j}}$ are the channel gains, $\alpha_{s}$ and $\alpha_{j}$ are the Nakagami- $m$ ditributed envelopes and $\theta_{s}$ and $\theta_{j}$ are the corresponding phases. Finally, in (2), $n(t)$ is the Additive White Gaussian Noise (AWGN) of one-sided power spectral density $N_{0}$. Note that for our subsequent investigations, we assume that all the users are simultaneously active and thus we have $U^{\prime}=U-1$.
The receiver schematic is shown in Fig. 2. The receiver is constituted by a bandpass filter, a frequency de-hopper, $M$ branches of the demodulator and finally a decision device. The bandpass filter removes any frequency that falls outside the spread spectrum signaling bandwidth. The de-hopper consists of a PN generator, which is identical to and aligned with the PN generator used in the transmitter shown in Fig. 1, a frequency synthesizer and a multiplier. The de-hopper de-spreads the recieved signal by exploiting the knowledge of the transmitter's unique FFH address. However, the de-hopping operation may result in spurious outputs, potentially imposing cochannel interference, owing to the fact that the PN FFH addresses of various users may not be perfectly orthogonal to each other. Each of the $M$ demodulator branches corresponds to an MFSK tone and consists of a square-law detector, followed by a diversity combiner, as shown in Fig. 2. The outputs of the square-law detectors may consist of the energy transmitted by the desired transmitter as well as that transmitted by the interfering users. At the end of the $L$ hop symbol interval, there are $M$ decision variables at the output of the diversity combiners, which we denote by $q_{m}, m=1,2, \ldots, M$. The detection decision is based on opting for the largest of the decision variables, i.e. the $k$ th MFSK tone is the transmitted one if $q_{k}$ is the maximum of $q_{m}, m=1,2, \ldots, M$. In the presence of MA interference, the energy received from the interfering users may lead to erroneous decisions and consequently, the BER of the system is severely degraded. However, FFH systems offer the advantage that each message symbol is transmitted in $L$ number of hops, thus resulting in beneficial diversity-assisted interference resilience. Therefore, the appropriate design of the diversity combiner, aimed at exploiting this diversity potential, is capable of reducing the degradation caused by interference. A number of diversity combiners will be discussed in Section III.

The spectral efficiency of the system under consideration is given by [2]

$$
\eta=\frac{U R_{b}}{W_{s s}}=\frac{U b R_{h} / L}{M / T_{h}}=\frac{U b}{M L} .
$$

\section{DIVERSITY COMBINING SCHEMES}

In this section we briefly discuss a variety of non-linear diversity combining methods that may be employed in FFH-MFSK based systems, in order to suppress the effects of MA interference. After the square-law detector stage of Fig. 2, the reciever has $M \times L$ variables for each transmitted symbol, corresponding to the $L$ hops and the $M$ tones. Let these variables be represented by $z_{m l}, m=1,2, \ldots M ; l=$ $1,2, \ldots L$. In the context of each of the diversity combining schemes, except in the case of product combining [7], the decision is based on the sum of a particular function of the outputs produced by the square-law detectors. Thus, the decision metric $q_{m}$ of a non-linear combining scheme is given by [7]

$$
q_{m}=\sum_{l=1}^{L} f\left(z_{m l}\right), \quad m=1,2, \ldots, M .
$$

It can be seen from (4) that in the context of linear combining, we have $f\left(z_{m l}\right)=z_{m l}[7]$.

\section{A. Hard Limiting Majority Vote Combining}

The conventional diversity combiner of the FFH-MA system proposed in [1] is referred to as the Hard Limiting Majority Vote (HLMV) combiner. In the context of this combining scheme, at the end of each hop, the output of a square-law detector is assigned a 
value of 1 , if it exceeds a pre-set threshold or 0 , if it does not. Hence, when employing the HLMV combiner, we have

$$
f\left(z_{m l}\right)= \begin{cases}1 & z_{m l} \geq \delta \\ 0 & \text { otherwise }, \quad m=1,2, \ldots M ; l=1,2, \ldots L\end{cases}
$$

where $\delta$ is a pre-set threshold. At the end of the $L$ hops, the assigned values are summed. Thus the decision variable corresponding to each FSK tone can be found by inserting $f\left(z_{m l}\right)$ given by (5) into (4).

\section{B. Self-Normalization Combining}

This diversity combining scheme is often also referred to as the Normalized Envelope Detection (NED) arrangement. When using this combining scheme, for each hop, the output of each square law detector seen in Fig. 2 is normalized by the sum of the outputs of all the $M$ detectors. The corresponding decision function is thus given by [6]:

$$
f\left(z_{m l}\right)=\frac{z_{m l}}{\sum_{m=1}^{M} z_{m l}}, \quad l=1,2, \ldots L .
$$

This normalization operation results in de-emphasizing of the interference energy. The normalized energy detector outputs are then summed over all hops and the decision variable corresponding to each hop is determined by substituting (6) into (4).

\section{Clipped Diversity Combining}

In the context of this technique [7] the outputs of the squarelaw detectors seen in Fig. 2 are clipped at a certain value, before they are combined for all hops. Hence, in the context of the Clipped Diversity Combining receiver, the function evaluated at the squarelaw detector's outputs can be expressed as

$$
f\left(z_{m l}\right)= \begin{cases}C & \text { for } z_{m l} \geq C \\ z_{m l} & \text { otherwise } \\ & m=1,2, \ldots M ; l=1,2, \ldots L .\end{cases}
$$

The final decision variable $q_{m}$ is determined by substituting (7) into (4) [7]. The value of $C$ in (7), which is usually referred to as the clipping threshold, is adjusted during each hop based on the estimate of the noise power and the number of active users. The philosophy governing this technique is that if more than one interfering user happens to activate the same MFSK tone during a hop interval, a high energy may be received in the corresponding branch of the demodulator. Thus, any energy received in excess of a certain level may be attributable to interference.

In [3], based on the maximum likelihood criterion, an optimum diversity combiner designed for FFH-MA systems has been derived, which operates on the principle of linearly combining a non-linear function of the energy detector outputs. Owing to the complexity of the derived combining scheme, it was suggested [3] that the optimum non-linearity may be approximated by a clipping function, as expressed in (7). This approximate optimum combiner, which is identical to the clipped diversity combiner discussed above, is termed as the Soft Limiting Combiner (SLC) in [3]. In the context of an FFHMA system, the SLC constitutes the best diversity combining scheme proposed in [3]-[6]. However, the SLC requires side information of the SNR and of the number of active users in order to accurately set the clipping threshold. The results of [4] demonstrate that even without accurate knowledge of the number of active users, the performance of the SLC is not significantly different from that achieved assuming perfect side information. However, again, the measurement of the noise power is mandatory in order to accurately adjust the clipping threshold.

\section{Product Diversity Combining}

As the terminology suggests, in the context of the Product Combining Receiver (PCR), the decision variable $q_{m}$ corresponding to each MFSK tone as seen in Fig. 2 is obtained by multiplying the outputs of the corresponding square-law detector for all hops, which can be expressed as [7]:

$$
q_{m}=\prod_{l=1}^{L}\left(z_{m l}\right), \quad m=1,2, \ldots M .
$$

The idea behind this technique is that the received signal corresponding to a non-signal tone will carry low power in at least one of the $L$ hops, with a high probability. Consequently, when the product of the detector outputs over all hops is computed, the decision variable $q_{m}$ corresponding to the non-signal tone yields a low value. By contrast, in the context of the desired signal tone, the product of the detector outputs generated for all hops will yield a significantly higher value, owing to the presence of the signal power in addition to any noise and jamming power. Thus, a correct decision is likely to be facilitated using this diversity combining scheme

\section{E. Order Statistics-Normalized Envelope Detection Based Diversity Combining}

In the context of Order Statistics (OS) based diversity combining [6], the $L$ outputs of each of the $M$ square law detectors are first ranked in ascending order of magnitude. Let the ranked square-law detector outputs $z_{m l}, m=1,2, \ldots M ; l=1,2, \ldots L$ as seen in Fig. 2 be represented by $z_{l m i}^{(r)}$, where $i, i=1,2, \ldots L$, represents the rank of that particular output in the ordered list. Thus, we have

$$
z_{l m 1}^{(r)} \leq z_{l m 2}^{(r)} \leq z_{l m 3}^{(r)} \leq, \ldots, z_{l m L}^{(r)}
$$

The basic idea behind the ordering is to identify the higher square-law detector outputs and thus to find ways of identifying the interference contaminated signals. Following this ranking, the self-normalization operation is performed on the ordered values as expressed in (6). Thus, the decision variable, in the context of the OS-NED is based on the function

$$
f\left(z_{m l}\right)=\frac{z_{l m i}^{(r)}}{\sum_{m=1}^{M} z_{l m i}^{(r)}}, \quad l=1,2, \ldots L,
$$

and is determined by substituting (9) into (4). The ordering and normalization result in stronger de-emphasis of the energy detector outputs corresponding to the interference-infested tones. Thus, the effects of interferences are substantially reduced.

\section{SIMULATION RESULTS AND DISCUSSION}

In this section we discuss the achievable performance of the various diversity combining schemes considered in Section III, when the FFH-MFSK system operates in a MA scenario and is subjected to Nakagami-m fading. It is customary to fix the hopping bandwidth to data rate ratio given by $W_{s s} / R_{b}$ and optimize the remaining parameters, i.e. $M$ and $L$, in order to achieve the best possible BER and $\eta$ [2], [6]. We assume having $W_{s s} / R_{b}=128$ for all subsequent investigations, unless otherwise stated. Hence, from (1) and from the assumption of $Q=M$, we have

$$
L=\frac{W_{s s}}{R_{b}} \frac{b}{M}
$$

Since, the objective of this contribution is to compare the performance of various diversity combining schemes, we arbitrarily opt for having 
$M=64$, which gives $L=12$ for $W_{s s} / R_{b}=128$. Note from (3) that, for a fixed $W_{s s} / R_{b}$, the spectral efficiency of the system depends only on the number of users supported.

In Fig. 3, we have plotted the BER against the total number of users for the diversity combining schemes outlined in Section III. The results portrayed in this figure correspond to $m=1$, which is equivalent to a Rayleigh fading channel, and $E_{b} / N_{0}=20 \mathrm{~dB}$, where $E_{b}$ is the transmitted energy per bit. The results of Fig. 3 show that the achievable performance of the linear combining scheme and the NED of Section III is poor, although when the number of users is low, for example when we have $U=10$, their BER curves drop relatively sharply. Moreover, Fig. 3 shows that the performance of the SLC is the best among the ones investigated, while the BER performance of the HLMV, the PCR and the OS-NED is similar to each other and inferior to that of the SLC.

In Fig. 4, the BER performance of the SLC, the HLMV, the PCR and the OS-NED based combining schemes is shown for two more $E_{b} / N_{0}$ values, namely for 10 and $15 \mathrm{~dB}$. It can be deduced from the results of Figs. 3 and 4 that the HLMV scheme is more sensitive to the SNR than the other schemes considered. Thus, as seen in Fig. 4 , for $E_{b} / N_{0}=10$ and $15 \mathrm{~dB}$ the performance of the HLMV is inferior to that of the PCR and the OS-NED, which is in contrast to the case when $E_{b} / N_{0}=20$ was assumed in Fig. 3. Moreover, for $E_{b} / N_{0}=10$ the performance of the OS-NED approaches that of the SLC.

The results of Fig. 5 show the effects of having different Nakagami fading parameters $m$ on the performance of the diversity combining schemes which were considered in Fig. (4). As expected, the performance of all the schemes is poor for $m=1.5$ and is better for $m=10$. Furthermore, Fig. 5 shows that the performance of the SLC is superior to those of the other schemes for all $m$ values, while that of the OS-NED is the next best.

In Fig. 6, the effects of increasing the diversity order on the performance of the SLC, the PCR and the OS-NED schemes are shown. With $M$ fixed at 64 , an increase in $L$ results in a corresponding increase in the $W_{s s} / R_{b}$ ratio. Thus, we have $W_{s s} / R_{b}=192$ and 256 for $L=18$ and 24 , respectively. As seen in Fig. 6 for increasing values of $L$, the performance of all the schemes improves and, for all $L$ values, the SLC results in the best performance among the schemes shown. Moreover, note that in Fig. 6 the OS-NED outperforms the PCR and approaches the performance of the SLC, when we have $L=24$.

In Fig. 7, the effects of increased modulation order, which implies an increase in the number of hopping tones owing to the assumption of $Q=M$, on the performance of three schemes is shown. In conjunction with $L=12$, an increase in $M$ results in a corresponding increase in the $W_{s s} / R_{b}$ ratio. Thus, we have $W_{s s} / R_{b} \approx 219$ and 384 for $M=128$ and 256 , respectively. As seen in Fig. 7 , for increasing values of $M$, the performance of all the schemes improves. This is primarily a benefit of a reduction in the probability of interference, when a high number of signalling tones is available. Note in Fig. 7 that the SLC significantly benefits from having increased $M$ and its BER curve drops well below those of the other two schemes shown for high number of users, for example when we have $U=50$ and 60. Moreover, Fig. 7 shows that the PCR outperforms the OS-NED for $M=128$ and 256.

In Fig. 8, the achievable BER corresponding to three diversity combiners is plotted against the SNR for $U=30$ users. The results of Fig. 8 indicate the significance of using optimum clipping thresholds in the context of the SLC. When a fixed threshold is employed in conjunction with the SLC for all SNR values, as seen in Fig. 8, the performance of the SLC degrades so much that even the PCR and the OS-NED outperform it for $E_{b} / N_{0}$ in excess of $22 d B$ or less than $13 d B$.

\section{CONCLUSION}

We have comparatively studied the performance of a range of diversity combining schemes in terms of their ability to combat MA interference, when they are used in a FFH-MFSK system communicating over Nakagami fading channels. Our simulation results demonstrate that the optimum SLC proposed in [3] outperforms all the other schemes considered. The linear and the NED based combining schemes offer little advantage in terms of suppressing the MA interference. Two other schemes, namely the OS-NED and the PCR, offer useful performance gains and, although their performance remains inferior in comparison to that of the optimum SLC, they constitute attractive design options. This is because, unlike the SLC, they do not require the assitance of side information. When a high diversity order is employed or when the SNR is relatively low, the performance of the OS-NED scheme approaches that of the SLC. Finally, in the absence of a reliable noise power estimate, the OSNED and the PCR outperform the SLC.

\section{REFERENCES}

[1] D. J. Goodman, P. S. Henry and V. K. Prabhu, "Frequency-hopped multilevel FSK for mobile radio", Bell System Technical Journal, 1980, 59 (7), pp. 1241-1255.

[2] U. C. Fiebig, "The efficiency of FFH/CDMA systems in a mobile radio environment", SUPERCOMM/ICC '94 Conference Record, Vol.1, pp. 525-529, May 1994.

[3] O. C. Yue, "Maximum likelihood combining for noncoherent and differentially coherent frequency hopping multiple access systems", IEEE Transactions on Information Theory, Vol. IT-28, NO. 4, pp. 631-639, July 1982.

[4] C. P. Hung and Y. T. Su, "Diversity combining considerations for incoherent frequency hopping multiple access systems", IEEE Journal on Selected Areas in Communications, Vol. 13, NO. 2, pp. 333-344, Feb. 1995.

[5] Y. T. Su and C. Y. Hsiao, "On the detection of a class of fat frequencyhopped multiple access signals", IEEE Journal on Selected Areas in Communications, Vol. 19, NO. 11, pp. 2151-2164, Nov. 2001.

[6] E. D. Spilchen and T. A. Gulliver, "Diversity combining for $M$-ary fast frequency hopping in a multiple access Rayleigh fading channel", Proceedings of MILCOM'96. IEEE Vol. 3, pp. 893-897, Oct. 1996.

[7] T. A. Gulliver, E. B. Felstead, R. E. Ezers and J. S. Wright, "A unified approach to time diversity combining of fast frequency hopped NCMFSK - anti-jam processing", Proceedings of MILCOM '94. IEEE Vol. 2 , pp. 415-420, Oct. 1994. 


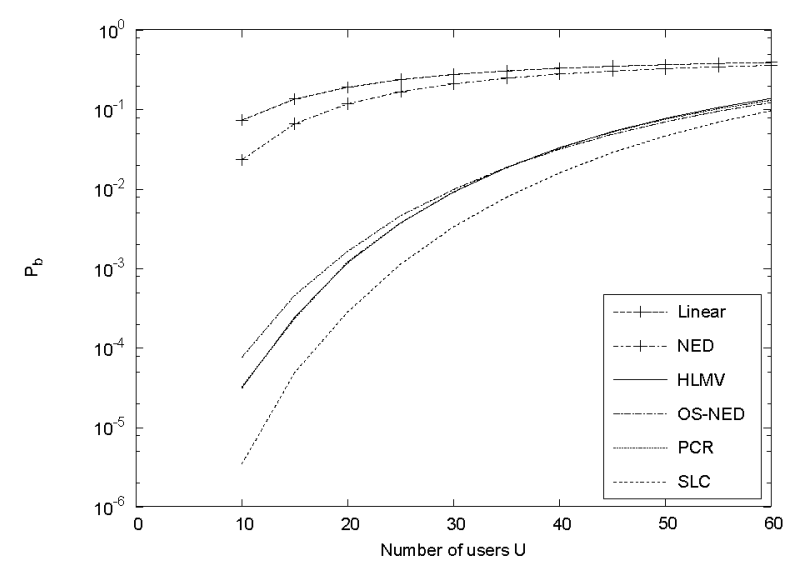

Fig. 3. The BER versus the number of users for various diversity combining schemes when employed in a FFH-MFSK system communicating over a Nakagami- $m$ fading multiple access channel, assuming $E_{b} / N_{0}=20 \mathrm{~dB}$, $L=12, M=64$ and $m=1$.

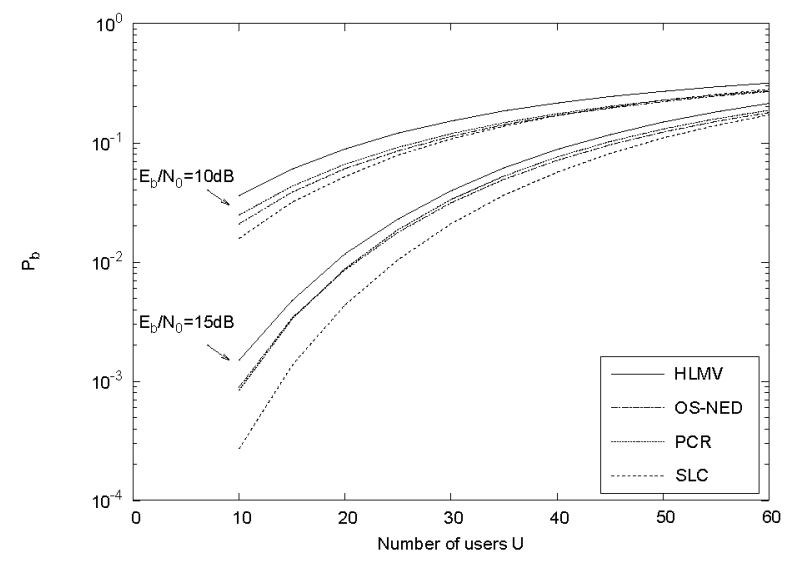

Fig. 4. The BER versus the number of users for various diversity combining schemes when employed in a FFH-MFSK system communicating over a Nakagami- $m$ fading multiple access channel, assuming $L=12, M=64$, $m=1$ and $E_{b} / N_{0}$ as a parameter.

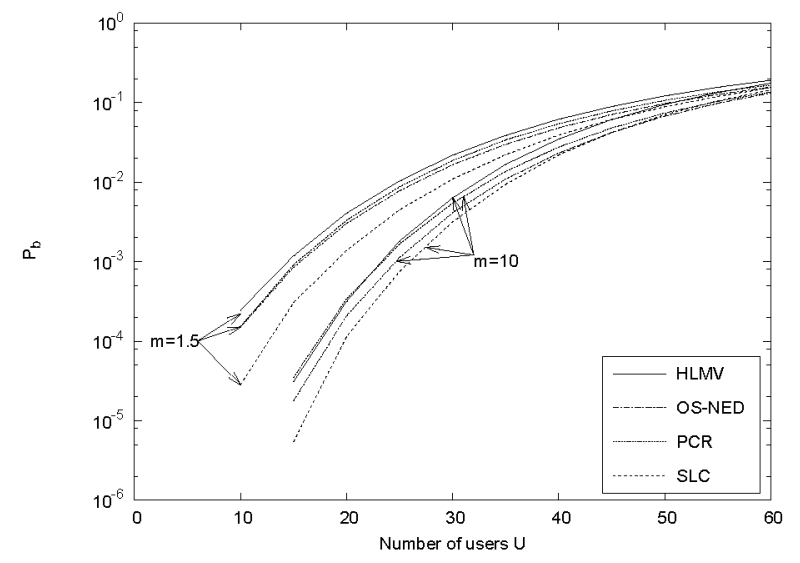

Fig. 5. The BER versus the number of users for various diversity combining schemes when employed in a FFH-MFSK system communicating over a Nakagami- $m$ fading multiple access channel, assuming $E_{b} / N_{0}=15 d B$, $L=12, M=64$ and $m$ as a parameter.

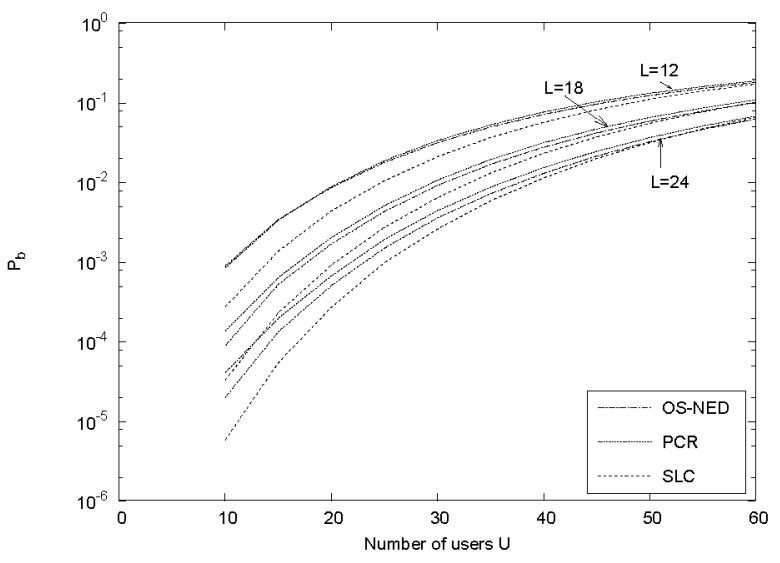

Fig. 6. The BER versus the number of users for various diversity combining schemes when employed in a FFH-MFSK system communicating over a Nakagami- $m$ fading multiple access channel, assuming $E_{b} / N_{0}=15 d B$, $m=1, M=64$ and $L$ as a parameter.

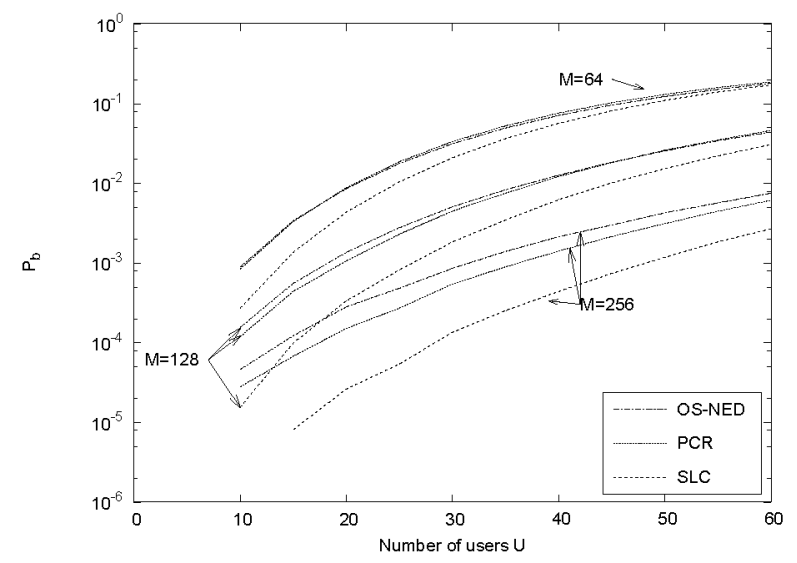

Fig. 7. The BER versus the number of users for various diversity combining schemes when employed in a FFH-MFSK system communicating over a Nakagami- $m$ fading multiple access channel, assuming $E_{b} / N_{0}=15 d B$, $L=12, m=1$ and $M$ as a parameter.

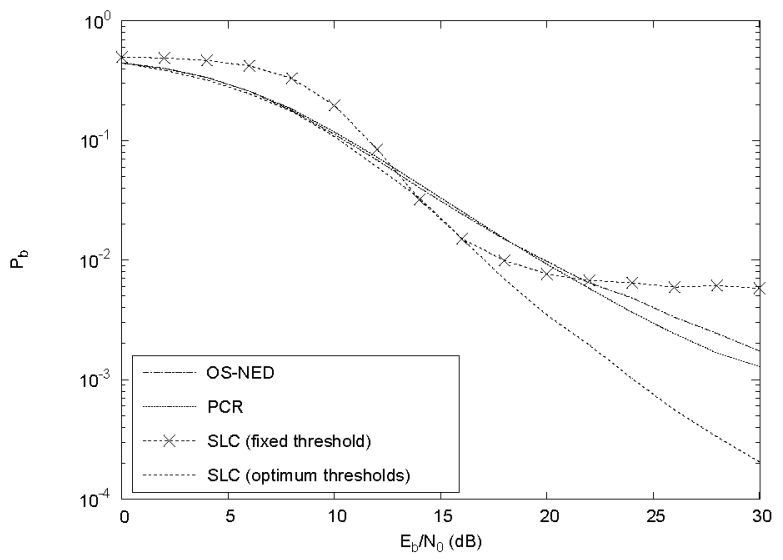

Fig. 8. The BER versus the SNR for various diversity combining schemes when employed in a FFH-MFSK system communicating over a Nakagami- $m$ fading multiple access channel, assuming $U=30, L=12, M=64$ and $m=1$. 